\title{
Estudo Morfológico e Morfométrico da Mama de Ratas em Estro Permanente, Tratadas com Danazol
}

\author{
Morphological and Morphometrical Study of Permanent Estrus Rat Mammary \\ Gland Treated with Danazol
}

Jorge Yoshinori Shida ${ }^{1}$, Luiz Henrique Gebrim ${ }^{1}$, Manuel Jesus Simões ${ }^{2}$, Edmund Chada Baracat ${ }^{1}$, Geraldo Rodrigues de Lima ${ }^{1}$

\begin{abstract}
RESUMO
Objetivo: estudar os efeitos do danazol sobre a mama da rata em estro permanente.

Métodos: os animais foram divididos em três grupos: os do grupo $A(n=12)$ receberam água (placebo), como grupo controle; os do grupo $B(\mathrm{n}=13)$ foram expostos a $20 \mathrm{mg} / \mathrm{kg}$ de danazol por dia; os do grupo $C(n=10)$ foram expostos a $80 \mathrm{mg} / \mathrm{kg}$ de danazol por dia. A droga foi administrada durante 35 dias consecutivos. O estudo microscópico dos cortes avaliou a distribuição ductal e acinar. A histometria da relação ducto/estroma foi feita baseada nos princípios da estereologia, com ocular $k-10 X$ da Zeiss, com retículo de integração (Integrationsplatte I-reticulo de Weibel de 25 hits), com aumento final de 100 vezes. Para cada lâmina estudada, foram contados 10 campos aleatórios, num total de 250 pontos. $O$ teste aplicado foi a análise de variância por postos de Kruskal-Wallis, para comparar os três grupos em relação ao número médio de alvéolos e ductos nas mamas $(\alpha=0,05)$.

Resultados: em todos os grupos, os lóbulos apresentaram ao estudo morfológico alvéolos revestidos com células cúbicas com núcleos na sua porção central ou basal. Pequenas quantidades de material eosinofilico foram observadas em alguns casos na sua luz. À morfometria (magnificação de 100X) encontraram-se em média 28,6 ductos/ 10 campos no grupo A, 28, 4 no grupo $B$ e 29,2 no grupo $C$ (análise de Kruskal-Wallis: $H_{\text {crit }}=0,1$ ). As médias dos números de alvéolos / 10 campos foram 5,9 (grupo A), 9,3 (grupo B) e 6,5 (grupo C) (Kruskal-Wallis $H_{\text {crit }}=$ 2.9), sem diferença significativa entre os grupos.

Conclusão: o danazol não causou nenhuma alteração significante na morfologia e morfometria do epitélio das mamas das ratas em estro permanente.
\end{abstract}

PALAVRAS-CHAVE: Danazol. Mama: doença benigna. Mastalgia. Modelos experimentais.

\section{Introdução}

A glândula mamária é uma estrutura que apresenta respostas variáveis aos hormônios esteróides. Sua interação hormonal é complexa, envolvendo os esteróides sexuais que atuam por

Trabalho realizado no Departamento de Ginecologia, e Disciplina de Histologia da UNIFESP-EPM, São Paulo.

${ }_{1}^{1}$ Disciplina de Ginecologia UNIFESP-EPM

2 Disciplina de Morfologia, UNIFESP-EPM

Correspondência:

Jorge Yoshinori Shida

Rua Leandro Dupré, 207 - Vila Clementino

04025-010 - São Paulo - SP

Tel.: 575-0171

e-mail: drshida@mobinet.com.br via autócrina, parácrina e endócrina. O estradiol possui importante papel no desenvolvimento das células ductais e do estroma. A progesterona, em sinergismo com o estradiol e a prolactina, promove o desenvolvimento e diferenciação acinar. As alterações dos hormônios esteróides podem determinar importantes distúrbios mamários como: dor, nodulações e neoplasias, tanto benignas quanto malignas ${ }^{1,2}$.

O danazol é uma das drogas preconizadas para o tratamento da dor e alterações funcionais benignas da mama por apresentar interessantes propriedades, com várias formas de atuação. Foi introduzido em terapêutica pela primeira vez por Greenblatt et al. ${ }^{3}$ em 1971 no tratamento da dor 
e das alterações fibrocísticas mamárias. Esses autores, naquela época, já indicavam a forma de ação terapêutica desse derivado isoxazólico da testosterona por meio do bloqueio gonadotrópico. Posteriormente, outros autores descreveram novas formas de ação, como: bloqueio da esteroidogênese e diminuição das proteínas carregadores de esteróides, além de outros mecanismos também importantes ${ }^{4,5}$. Atualmente, Ting et al. ${ }^{6}$ estenderam o uso de danazol para tratamento clínico de ginecomastia. Apesar destes ensaios clínicos, sabe-se pouco sobre a ação desta droga em nivel celular na mama ${ }^{7-9}$.

Devido à dificuldade de estudar a glândula mamária humana normal pelas restrições éticas, o modelo experimental surge como boa opção para pesquisa. Dentre os modelos, o animal que se assemelha à mulher em relação ao estudo endócrino e mamário é a rata. Seu ciclo endócrino (estral) foi importante para a compreensão do ciclo menstrual. Além disso, sua morfologia mamária apresenta formação de ductos e alvéolos como nas mulheres. Apresenta, também, comportamento citogenético simi$\operatorname{lar}^{10-12}$.

O estado de estro permanente apresenta características interessantes para o estudo hormonal e mamário. Caracteriza-se pela falta de atividade reprodutiva cíclica determinando anovulação crônica, infertilidade e ovários policísticos, e na mama, notam-se hipertrofia ductal e alveolar, secreção no interior de sua luz e sinais indiretos de atrofia do estroma, o que facilita a observação das alterações inibitórias sobre os ductos ${ }^{13,14}$.

Várias técnicas induzem o estro permanente: a gonadectomia e transplante testicular em ratas recém-natas, exposição contínua à luz, exposição a esteróides sexuais na fase crítica do desenvolvimento do animal, ablação do núcleo supraquiasmático, etc. No entanto, a forma mais simples foi desenvolvida por Barraclough ${ }^{15}$, por meio da injeção de propionato de testosterona (125 mg) via subcutânea na rata recém-nascida até o 5o dia de vida. Esta exposição androgênica determina profundas alterações na função ovariana e de outros órgãos, devido a mudanças irreversiveis no hipotálamo durante o estádio crítico de seu desenvolvimento ${ }^{6,14}$. A condição anovulatória determina elevação crônica de estrogênio, sendo máxima nestas ratas no período de 60 a 185 dias $^{16,17}$. A rata androgenizada possui níveis constantes de estrogênio e, como não ovula, não há progesterona. Os ovários são policísticos, a demonstrar o efeito de níveis de LH e FSH, apesar de haver algum bloqueio enzimático ${ }^{18}$.
A exposição continua ao estrogênio determina alterações na morfologia mamária, como mamilos proeminentes, hipertrofia dutal e acinar com secreção eosinofílica na luz e discutivel hipertrofia do estroma. Este meio estimulado nos parece ser adequado para avaliar drogas com ações antiestrogênicas, como realizado por Alcântara ${ }^{12}$ utilizando o tamoxifeno. É interessante o estudo de droga que apresente ação hipotalâmica central e também periférica, para testar seus efeitos sobre a mama na condição anovulatória e estrogênica.

Assim, propusemo-nos a estudar as alterações morfológicas e morfométricas do epitélio das mamas de ratas em estro permanente submetidas a diferentes doses de danazol.

\section{Material e Métodos}

Utilizaram-se ratas (Rattus norvegicus albinus, Rodentia Mammalia) EPM-I Wistar que foram induzidas a estro permanente por meio de injeção de propionato de testosterona, $125 \mathrm{mg}$, subcutâneo, na região dorso-cervical, até o terceiro dia de vida ${ }^{6}$.

Por meio da citologia seriada durante uma semana, selecionaram-se apenas as ratas adultas (três meses de vida) que apresentavam cornificação persistente da vagina, comprovando o estado de estro permanente.

Os animais foram mantidos em gaiolas plásticas com grade de metal, alimentados com ração padrão (Labina-Purina, São Paulo, Brasil) e água ad libitum, na temperatura ambiente de $22^{\circ} \mathrm{C}$, com iluminação artificial produzida por lâmpada fluorescente de 40 watts, sendo o fotoperíodo de luz das 7:00 às 19:00 horas.

Os animais foram divididos em três grupos: grupo A (controle) $(n=12)$, grupo $B(n=13)$ e grupo $C(n=10)$. As ratas do grupo A foram submetidas a $4 \mathrm{ml}$ de água, via oral, as do grupo $\mathrm{B}$ a $20 \mathrm{mg} / \mathrm{kg}$ por dia de danazol e as do grupo C a $80 \mathrm{mg} / \mathrm{kg}$ por dia, de danazol por via oral (método de gavagem), durante 35 dias. Para tanto, usou-se o conteúdo das cápsulas de danazol de $50 \mathrm{mg}$ e $200 \mathrm{mg}$, diluído em $50 \mathrm{ml}$ de água, obtendo-se a emulsão de $1 \mathrm{mg} / \mathrm{ml} \mathrm{e} 4 \mathrm{mg} / \mathrm{ml}$, respectivamente.

No $36^{\circ}$ dia, todos os animais foram sacrificados, sob anestesia inalatória com éter etílico; através de abertura da pele e do subcutâneo na linha média abdominal, dissecou-se a área correspondente a duas unidades glandulares abdômino-inguinais à direita, que foram fixadas em formol a $10 \%$ e processadas em parafina. Obti- 
veram-se, então, as lâminas com espécimes padronizados de $4 \mu \mathrm{m}$ coradas por hematoxilinaeosina ${ }^{10}$.

Procurou-se com estudo microscópico dos cortes a avaliação da distribuição ductal e acinar, assim como o padrão secretor. Utilizou-se microscópio de luz da marca Carl-Zeiss. A histometria da relação ducto/estroma foi feita baseada nos princípios da estereologia, de acordo com Weibel et al. ${ }^{19}$. No estudo utilizou-se ocular k 10X da Zeiss, com retículo de integração (Integrationsplatte I), contendo 25 pontos dispostos simetricamente em cinco fileiras de cinco pontos cada (retículo de Weibel de 25 hits), acoplado em microscópio de luz com aumento final de 100 vezes. Para cada lâmina estudada, contaram-se 10 campos aleatórios pelo sistema de varredura horizontal, num total de 250 pontos.

Foi aplicada a análise de variância por postos de Kruskal-Wallis, com a finalidade de comparar os três grupos em relação ao número médio de alvéolos e ductos nas mamas, da média de 10 campos, com aumento de 100 vezes $(\alpha=$ $0,05)^{20}$.

\section{Resultados}

A histologia das glândulas mamárias de ratas do grupo A (controle) evidenciou lóbulos, ductos e tecido conjuntivo interlobular. Os lóbulos apresentaram alvéolos revestidos por células cúbicas, contendo núcleo esférico em sua porção central ou basal. Na sua luz observou-se em alguns casos material eosinófilo em pequena quantidade, principalmente no grupo A. Circundando os lóbulos, encontramos grande concentração de tecido adiposo, constituído por células esféricas, com citoplasma claro e núcleo alongado, deslocado para a periferia. Os ductos eram representados por células achatadas, com núcleo elíptico, contendo, às vezes, o mesmo material eosinófilo dos alvéolos. Eram, em geral, envoltos por tecido conjuntivo denso (Figura $1)$.

De permeio ao tecido adiposo observaramse regiões de tecido conjuntivo denso, formado por células fusiformes, citoplasma escasso (fibrócitos) e fibras colágenas.

$\mathrm{O}$ estudo da mamas de ratas medicadas com o danazol indicou que tanto no grupo B quanto no $\mathrm{C}$, podiam-se observar características semelhantes às do grupo controle.

O grupo A (controle) apresentou a média de 28,6 ductos / 10 campos, ao passo que os grupos B e C não apresentaram alteração significativa no número de ductos quando comparados entre si, sendo as médias, respectivamente, 28,4 e 29,2 ductos / 10 campos (Tabela 1 ).

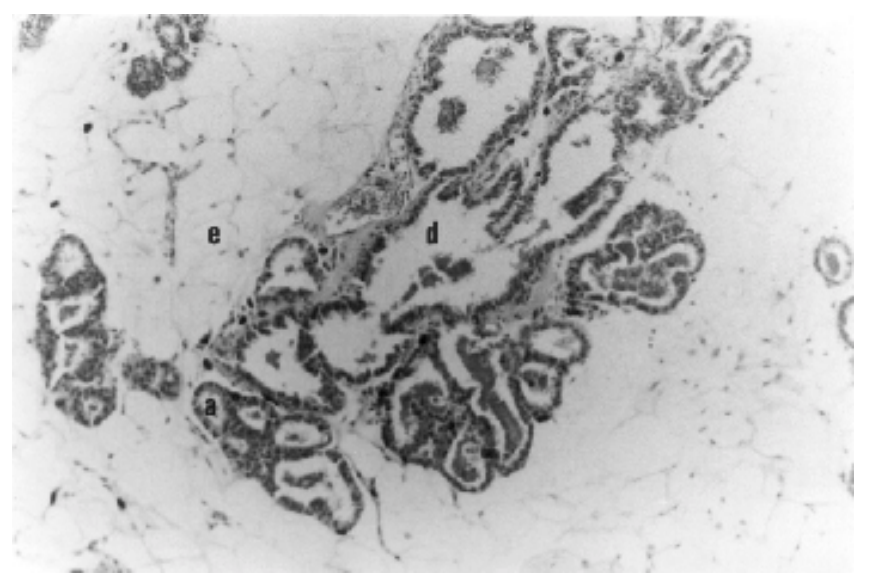

Figura 1 - Fotomicrografia mostrando uma parte da mama de rata em estro permanente tratado com água (grupo controle). Observaram-se os ductos (d), alvéolos (a), secreção intraluminar e o estroma (e). HE (140X).

Tabela 1 - Número médio de alvéolos nos grupos A (controle), B (20 mg danazol kg-1 dia 1) e C (80 mg danazol kg-1 dia-1) em 10 campos, com aumento de 100 vezes.

\begin{tabular}{cccc}
\hline Rata & Grupo A & Grupo B & Grupo C \\
\hline 1 & 5 & 4 & 6 \\
2 & 6 & 7 & 9 \\
3 & 3 & 18 & 12 \\
4 & 5 & 5 & 6 \\
5 & 2 & 7 & 10 \\
6 & 1 & 5 & 5 \\
7 & 10 & 14 & 4 \\
8 & 7 & 8 & 7 \\
9 & 9 & 5 & 3 \\
10 & 8 & 18 & 3 \\
11 & 7 & 10 & - \\
12 & 8 & 13 & - \\
13 & - & 7 & - \\
Média & 5,9 & 9,3 & 6,5 \\
\hline
\end{tabular}

Análise de variância por postos de Kruskal-Wallis

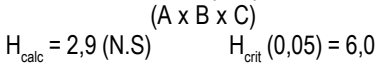

O número de alvéolos nos grupos $\mathrm{B}$ e $\mathrm{C}$, respectivamente 9,3 e 6,5 alvéolos/10 campos, não variou significativamente em relação ao grupo controle (Tabela 2). 
Tabela 2 - Número médio de dutos nos grupos A (controle), B (20 mg danazol kg-1 dia1) e C (80 mg danazol kg-1 $\left.\mathrm{dia}^{-1}\right)$, em 10 campos com aumento de 100 vezes.

\begin{tabular}{cccc}
\hline Rata & Grupo A & Grupo B & Grupo C \\
\hline 1 & 18 & 27 & 42 \\
2 & 30 & 31 & 22 \\
3 & 27 & 29 & 29 \\
4 & 20 & 25 & 28 \\
5 & 22 & 23 & 34 \\
6 & 25 & 21 & 20 \\
7 & 32 & 29 & 25 \\
8 & 18 & 17 & 35 \\
9 & 47 & 25 & 28 \\
10 & 33 & 44 & 29 \\
11 & 30 & 28 & - \\
12 & 41 & 33 & - \\
13 & - & 37 & - \\
Média & 28,5 & 28,3 & 29,2 \\
\hline
\end{tabular}

Análise de variância por postos de Kruskal-Wallis

$\mathrm{H}_{\text {calc }}=0,1$ (N. S.) $\quad \mathrm{H}_{\text {crit }}(0,05)=6,0$

\section{Discussão}

Nosso estudo foi motivado pela ampla utilização do danazol na terapêutica da dor mamária cíclica e das alterações fibrocísticas, e por se conhecer muito pouco do seu efeito direto sobre o lóbulo mamário normal ${ }^{21}$.

A dificuldade de avaliar os efeitos das drogas antiestrogênicas em mamas humanas, por problemas éticos, estimulou-nos a utilizar a rata, cuja morfologia mamária assemelha-se à da mulher. Para avaliar os possiveis efeitos antiestrogênicos do danazol sobre a mama da rata, imaginou-se um parênquima previamente estimulado pelo estrogênio e encontrou-se essa particularidade no modelo experimental de ratas em estro permanente ${ }^{6,10,13}$.

De fato, nestas ratas, como mostrou Sou$\mathrm{za}^{10}$, há desenvolvimento da unidade lóbuloacinar com secreção intraluminar (atividade secretora) e nítido predomínio dos dutos sobre os alvéolos, possivelmente resultante da estimulação crônica pelos estrogênios. Estes fatores tornam este modelo experimental interessante para se verificar o efeito do danazol sobre o epitélio mamário.

Nosso objetivo foi avaliar o efeito direto do danazol sobre o parênquima mamário, com a dose preconizada em ratas, tentando estabelecer uma equivalência com a dose tolerada pelo ser humano (1 g e $4 \mathrm{~g})^{10}$. Reconhece-se, contu- do, a limitação em extrapolar os resultados para o ser humano, pois o modelo utilizado é bastante específico e talvez se assemelhe à mulher com a síndrome anovulatória crônica. O período de exposição à droga foi de 35 dias, ou seja, aproximadamente seis a sete ciclos estrais, correspondendo a seis ou sete meses em mulheres.

Muito se discutiu sobre o verdadeiro mecanismo pelo qual o danazol produz atrofia dos focos de endometriose e regressão clínica das alterações cíclicas da mama, se por meio da inibição gonadotrópica ou por efeito local. O efeito do isoxazol sobre o eixo hipotálamo-hipofisário depende, em grande parte, do modelo animal escolhido para o estudo. Diferenças de espécies, de idade, sexo e estado gonadal podem levar a resultados variados ${ }^{22}$.

A ação sobre os receptores estrogênicos é duvidosa e alguns autores acreditam que o efeito do danazol sobre os receptores seja dependente da dose, duração e tecido em estudo. Por outro lado, o uso da droga faria diminuir os níveis de LH e FSH, e bloquearia a esteroidogênese ovariana por meio da inibição direta de várias enzimas como a 17 - $\alpha$-hidroxilase e a 3 - $\beta$ hidroxiesteróido-desidrogenase ${ }^{23,24}$.

Os resultados levam-nos a discutir alguns pontos interessantes do mecanismo de ação do danazol sobre mamas de ratas em estro permanente. A droga distorce o funcionamento do hipotálamo e da hipófise, acarretando anovulação. $\mathrm{Na}$ rata em estro permanente parece não haver esse efeito, uma vez que a anovulia é pré-existente e os animais continuaram em estro durante a administração da droga, confirmado pelo estudo anatomopatológico da vagina. Portanto, se houvesse algum efeito do fármaco sobre o epitélio da mama, esse deveria ser direto.

Verificou-se discreto predominio de material eosinofilico no grupo controle em relação aos grupos submetidos ao danazol; seria um efeito inibidor sobre a secreção ducto-alveolar, entretanto este dado não foi avaliado objetivamente. Futuros estudos poderão confirmar esta observação e outros, utilizando tecido mamário humano, respeitando as restrições éticas, poderão ser testados com intuito de avaliar a ação direta da droga sobre o tecido mamário.

Conclui-se que, nas doses empregadas $(20$ e $80 \mathrm{mg} \mathrm{kg}^{-1} \mathrm{dia}^{-1}$ ), o epitélio mamário foi indiferente ao danazol. Pode-se admitir que nestas doses a inibição da esteroidogênese por um lado e a testosterona livre, conseqüente à suposta queda de SHBG, por outro, não alcançaram um nível crítico necessário para inibir a produção dos estrogênios no parênquima mamário ou deslocálos de seu receptor. 


\section{SUMMARY}

Purpose: the morphologic and morphometric aspects of the breasts of rats in permanent estrus submitted to danazol were studied.

Methods: the animals were divided into three groups: group $A(n=12)$ received water and was used as control, group $B(n$ =13) was exposed to $20 \mathrm{mg}^{\text {danazol }} \mathrm{kg}^{-1}$ day $\mathrm{y}^{-1}$ and group $C$ $(\mathrm{n}=10)$ was exposed to $80 \mathrm{mg}$ danazole $\mathrm{kg}^{-1}$ day-1 for 35 consecutive days. The microscopic study evaluated the ductal and acinar distribution. Histometry of the relationship duct/ stroma was based on the principles of stereology with a Zeiss $k$-10X ocular, with Integrationsplatte I reticulum of Weibel of 25 hits, with $100 X$ magnification. For each studied section, 10 aleatoric fields were counted, with a total of 250 points. The variance analysis test (Kruskal-Wallis) was applied to compare the three groups in relation to the mean number of alveoli and ducts $(\alpha=0.05)$.

Results: when submitted to morphological study, all groups presented lobules with alveoli lined with cubic cells with nuclei in their central or basal portion. Small amounts of eosinophilic material were observed in some cases in the lumen, with no differences between the groups. At morphometry, with a magnification of $100 X$, a mean number of 28.6 ducts/10 fields was found in group $A, 28.4$ in group $B$ and 29.2 in group $C$ (Kruskal-Wallis test: $H_{\text {crit }}=0.1$ ). The mean number of alveoli in 10 fields was 5.9, 9.3 and 6.5 in groups $A, B$ and $C$, respectively (Kruskal-Wallis test: $H_{\text {crit }}=2.9$ ), with no significant differences between the groups.

Conclusion: danazol did not cause any changes in the morphology and morphometry of the permanent estrus mammary epithelium.

KEY WORDS: Danazol. Breast: benign disease. Mastalgia

\section{Referências}

1. Humphrey LJ, Estes NC. Aspects of fibrocystic disease of the breast. Treatment with danazol. Postgrad Med J 1979; 55 Suppl 5: 48-51.

2. Chamness GC, Asch RH, Pauerstein CJ. Danazol binding and translocation of steroid receptors. Am J Obstet Gynecol 1980; 136:426-9.

3. Greenblatt RB, Dmowski WP, Mahesh VB, Scholer HF. Clinical studies with an antigonadotropin - danazol. Fertil Steril 1971; 22:102-12.

4. Barbieri LR, Canick JA, Makris A, Todd RB, Davies IJ, Ryan KJ. Danazol inhibits steroidogenesis. Fertil Steril 1977; 28:809-13.

5. Steinbrunn BS, Zera RT, Rodriguez JL. Mastalgia. Tailoring treatment to type of breast pain. Postgrad Med J 1997; 102:183-4, 187-9,193-4.

6. Ting AC, Chow LW, Leung YF. Comparison of tamoxifen with danazol in the management of idiopathic gynecomastia. Am Surg 2000; 66:38-40.

7. Barraclough C, Gorski RA. Evidence that hypothalamus is responsible for androgen-induced sterility in the female rat. Endocrinology 1961; 68:68-79.

8. Pinotti JA, Bedone AJ, Alvarenga M. Displasias mamárias: enfoque global. In: Pinotti JA, editor. Compêndio de Mastologia. $1^{a}$ ed. São Paulo: Manole; 1991. p.203-18.

9. McFayden IJ, Forrest AP, Chetty U, Raab G. Cyclical breast pain: some observations and the difficulties in treatment. Br J Clin Pract 1992; 46:161-4.

10.Souza AZ. Contribuição para o estudo da mama de ratas em estro permanente, em hipotireoidismo experimental [dissertação]. São Paulo: Universidade Estadual de São Paulo; 1970.

11.Russo J, Russo I H. Biological and molecular bases of mammary carcinogenesis. Lab Invest 1987; 57:112-37.

12.Alcantara AMPT. Estudo morfológico e morfométrico da glândula mamária de ratas em estro permanente tratadas com tamoxifeno [dissertação]. São Paulo: Universidade Federal de São Paulo; 1996.

13.Rodrigues de Lima G. Contribuição para o estudo da fisiopatologia de ratas em estro permanente: sensibilidade uterina à ocitocina [tese]. São Paulo: Universidade Federal de São Paulo; 1966.

14.Singh KB. Persistent estrus: an experimental model of the polycystic ovary syndrome. Obstet Gynecol Surv 1969; 24:2-17.

15.Barraclough CA. Induction of sterility in the female rat bay single injections of testosterone propionate. Anat Rec 1958; 130:267-72.

16. Chiórboli E. Contribution to study in vitro steroid hormones secretion by ovary of normal rats and testosterone treated rats [dissertação]. São Paulo: Universidade Federal de São Paulo; 1970.

17. Matt DW, Tedeschi ML, Sayles TE. Alterations in gonadotropin secretion in middle-aged persistentestrous rats following LHRH-agonist treatment. Neuroendocrinology 1992; 56:780-7.

18.Pasqualini JR, Chetrite G, Nguyen BL, et al. Estrone sulfate-sulfatase and 17 beta-hydroxysteroid dehydrogenase activities: a hypothesis for their role in the evolution of human breast cancer from hormonedependence to hormone-independence. J Steroid Biochem Mol Biol 1995; 53:407-12.

19.Weibel ER, Kistler GS, Scherle WF. Practical stereological methods for morphometric cytology. J Cell Biol 1966; 30:23-37.

20.Siegel S. Estadística no paramétrica aplicada a las ciencias de la conducta. $1^{a}$ ed. México: Editorial Trillas; 1975. p.346.

21.Rodrigues de Lima G, Gebrim LH. Displasia mamária: revisão das drogas empregadas no seu tratamento. Rev Paul Med 1986;104:82-6.

22.Potts GO. Pharmacology of danazol. J Int Med Res 1977; 5 Suppl 3:1-17.

23.Taguchi M, Kubota T, Aso T. Direct effect of danazol on the DNA synthesis and ultrastructure of human cultured endometrial stromal cells. Gynecol Obstet Invest 1995; 39:192-6.

24.Baracat FF. Estudo histométrico de pacientes com displasia mamária tratadas com várias drogas [dissertação]. São Paulo: Universidade Federal de São Paulo; 1990. 